\title{
Study of the Antifungal Action of the Lacquer Based on the GABA Derivative of 2-Chloro- $N$-(9,10-Dioxo-9,10- Dihydroanthracen-1-yl)Acetamide
}

\author{
Maryna Stasevych ${ }^{1, *}$ (D), Viktor Zvarych ${ }^{1}$ (D), Volodymyr Novikov ${ }^{1}$ (D) \\ 1 Department of Technology of Biologically Active Substances, Pharmacy and Biotechnology, Lviv Polytechnic National \\ University, 13, 79013 Lviv, Ukraine \\ * Correspondence: maryna.v.stasevych@lpnu.ua;
}

Scopus Author ID 8636372500

Received: 17.07.2020; Revised: 15.08.2020; Accepted: 16.08.2020; Published: 21.08.2020

\begin{abstract}
Onychomycosis is one of the most acute problems of modern medicine, which accounts for about 50\% of all nail diseases. Fungi of dermatophytes of the genus Trichophyton are the most studied etiological factor of onychomycosis. Non-dermatophytes can also cause onychomycosis. Fungi of the genus Aspergillus is one of the main pathogens among the non-dermatophytes agents of onychomycosis. Antifungal nail lacquer is the most optimal dosage form for the topical treatment of onychomycosis. GABA derivative of 2-chloro-N-(9,10-dioxo-9,10-dihydroanthracen-1-yl)acetamide was used as a perspective antifungal agent in the form of lacquer. The antifungal action of the obtained lacquer containing compound 2 against fungus strain Aspergillus niger VKM F-1119 was compared with the known antifungal lacquer "Lamisil" containing terbinafine as the active agent. Investigation of the antifungal action was carried out at concentrations of $1 \%, 0.5 \%$, and $0.1 \%$ of compound 2 and terbinafine using the diffusion in agar technic after $24 \mathrm{~h}$ and $72 \mathrm{~h}$. It has been determined that derivative 2 , as well as terbinafine at a concentration of $1 \%$, caused fungicidal action after $24 \mathrm{~h}$ and fungicidal and fungistatic effect after $72 \mathrm{~h}$.
\end{abstract}

Keywords: lacquer; antifungal action; Aspergillus niger; GABA; 2-chloro- $N$-(9,10-dioxo-9,10dihydroanthracenyl)acetamide.

(C) 2020 by the authors. This article is an open-access article distributed under the terms and conditions of the Creative Commons Attribution (CC BY) license (https://creativecommons.org/licenses/by/4.0/).

\section{Introduction}

Onychomycosis is one of the most acute problems of modern medicine, which accounts for about 50\% of all nail diseases [1-3]. Fungi of dermatophytes of the genus Trichophyton are the most studied etiological factor of onychomycosis [4]. Non-dermatophytes - yeast and mold fungi can also cause onychomycosis [5]. Fungi of the genus Aspergillus is one of the main pathogens among the non-dermatophytes agents of onychomycosis [6-8].

Antifungal nail lacquer is the most optimal dosage form for the topical treatment of onychomycosis [9]. They are devoid of some serious disadvantages (for example, the active substance is not fixed on the nail plate for a long time; the unsightly appearance of the nail plate when applying a cream or ointment; a long period of use of the drug, the multi-stage process of application, etc.) compared to such dosage forms in the form ointments, creams, and solutions $[10,11]$. 
The medicaments in the form of nail lacquers [12-14] are developed with physicochemical and physiological characteristics of the nail, so they can be applied directly to the nail plate. A protective film forms on the surface of the nail, helping to prevent the flow of oxygen from the outside and the mycotic microorganisms spreading [15]. Also, due to this film, the concentration of medicinal substances inside the nail remains high. Most antifungal nail lacquers not only treat the disease but also improve the condition of the nail plates [16, 17]. It should be noted that some lacquers, which include amino acids (lysine, methionine) have pronounced regenerative properties $[18,19]$. Amino acids allow to strengthen nails, improve its protective properties, and the ability to resist fungal infections [18, 19].

Derivatives of 9,10-anthracenedione are promising objects for the search for new antimicrobial, including antifungal agents [20-26]. Previously [22], we had synthesized a series of amino acid derivatives of 2-chloro- $N$-(9,10-dioxo-9,10-dihydroanthracen-1-yl)acetamide, among which GABA derivative with antifungal effect against the test culture of the fungus $A$. niger VKM F-1119 at a minimum inhibitory concentration of $1.9 \mu \mathrm{g} / \mathrm{ml}$ was selected.

Therefore, the aim of this study was to obtain the lacquer based on GABA derivative of 2-chloro- $N$-(9,10-dioxo-9,10-dihydroanthracen-1-yl)acetamide and to study its antifungal effect against the strain $A$. niger in comparison with the known antifungal lacquer.

\section{Materials and Methods}

\subsection{Materials.}

4-((2-((9,10-Dioxo-9,10-dihydroanthracen-1-yl)amino)-2-oxoethyl)amino)butanoic acid 2 was obtained from 2-chloro-N-(9,10-dioxo-9,10-dihydroanthracen-1-yl)acetamide 1 according to the described method [20]. All chemicals were of reagent grade and used without further purification. The solvents were purified according to the standard procedures [27].

Colorless nail lacquer "Nogotok" (Cosmetic-Service, Ukraine) was used as a basis for the preparation of antifungal lacquer with compound 2. Antifungal nail lacquer "Lamisil 1\%" (manufacturer "Delpharm Uning SAS", France) was used as a reference drug.

\subsection{Chemistry.}

The melting points were measured on a Boetius melting point-device and are uncorrected. ${ }^{1} \mathrm{H}$ NMR (400 MHz) and ${ }^{13} \mathrm{C}$ NMR (100 MHz) spectra were recorded in DMSOd6 solutions on a Varian Mercury-400 spectrometer with TMS as an internal standard. IR spectra were recorded in $\mathrm{KBr}$ pellets on the Specord M-80 spectrometer. Mass spectra were recorded on an Agilent 1100 Series G1956BLC/MSD SL LCMS system using electrospray ionization at atmospheric pressure $(70 \mathrm{eV})$. Elemental analysis was performed on a PerkinElmer CHN-analyzer Series 2400. The individuality of the obtained compound was monitored by TLC on Silufol UV-254 plates.

$$
\text { 4-((2-((9,10-Dioxo-9,10-dihydroanthracen-1-yl)amino }) \text {-2-oxoethyl)amino }) \text { butanoic }
$$

acid 2.

Yield 73\%. M.p. $=190-191{ }^{\circ} \mathrm{C} .{ }^{1} \mathrm{H}$ NMR, $\delta$, ppm: $1.85\left(\mathrm{~m}, 2 \mathrm{H}, \mathrm{CH}_{2}\right), 2.46(\mathrm{~m}, 2 \mathrm{H}$, $\mathrm{CH}_{2}$ ), 3.47 (m, H, CH 2$), 4.11$ (m, 2H, $\mathrm{CH}_{2}$ ), 4.61 (s, 1H, NH), 7.86-7.79 (m, 3H, $\mathrm{CH}_{\mathrm{ar}}$ ), 8.318.13 (m, 3H, CHar), 9.11 (d, 1H, J = $7.7 \mathrm{~Hz}, \mathrm{CH}_{\mathrm{ar}}$ ), 10.72 (s, 1H, NH), 12.85 (s, 1H, OH). IR, $\mathrm{sm}^{-1}: 3423\left(\mathrm{CH}_{2} \mathrm{NH}\right), 3368(\mathrm{NHCO}), 3000-2500(\mathrm{COOH}), 1721(\mathrm{C}=\mathrm{O}$ in $\mathrm{COOH}), 1685,1627$ 
$(\mathrm{C}=\mathrm{O}), 1643$ (CONH). LC-MS, m/z (Irel, \%): 367 [M+1] (100\%). Found, \%: C 65.49; H 5.03; N 7.71. $\mathrm{C}_{20} \mathrm{H}_{18} \mathrm{~N}_{2} \mathrm{O}_{5}$. Calculated, \%: C 65.57; $\mathrm{H} 4.95 ; \mathrm{N} 7.65$.

\subsection{Antifungal action of the lacquer.}

Antifungal effect of the lacquer based on 4-((2-((9,10-dioxo-9,10-dihydroanthracen-1yl)amino)-2-oxoethyl)amino)butanoic acid 2 at concentrations of $0.1 \%, 0.5 \%$ and $1 \%$ was investigated by diffusion in peptone on nutrient medium (wort agar) [28]. The microbial loading was $10^{9}$ cells (spores) $/ \mathrm{cm}^{3}$. The required incubation period were $48-72 \mathrm{~h}$ at $28-30{ }^{\circ} \mathrm{C}$. The results were recorded by measuring the zones surrounding the disk. Reference disk contained «Lamisil» at concentrations of $0.1 \%, 0.5 \%$ and $1 \%$. The control disk contained lacquer without antifungal agents.

\section{Results and Discussion}

\subsection{Chemistry.}

Continuing our research on the search for new substances with practically valuable properties among the functionalized derivatives of 9,10-anthraquinone [29-33], in work presented herein we report a study of the antifungal action of the lacquer based on the 4-((2((9,10-dioxo-9,10-dihydroanthracen-1-yl)amino)-2-oxoethyl)amino)butanoic acid 2 obtained using the method [20] (Scheme 1).

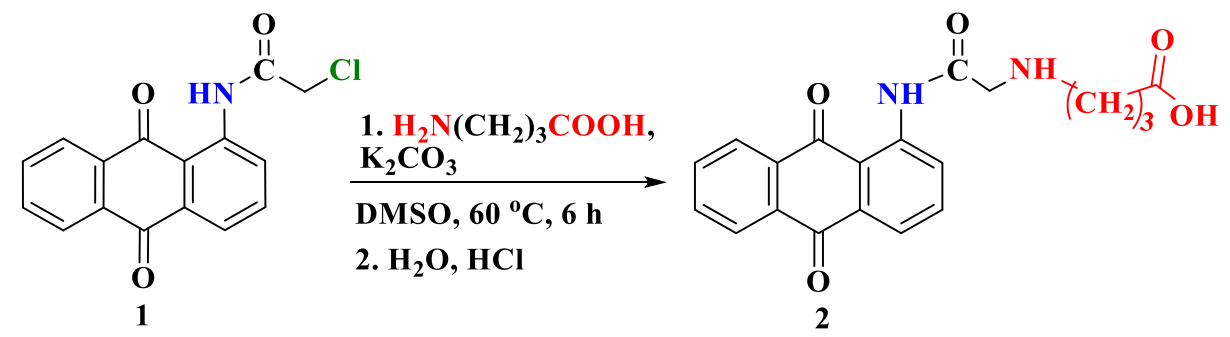

Scheme 1. Synthesis of 4-((2-((9,10-dioxo-9,10-dihydroanthracen-1-yl)amino)-2-oxoethyl)amino)butanoic acid 2.

\subsection{Investigation of antifungal action.}

The colorless nail lacquer «Nogotok» containing the following components: ethyl acetate, butyl acetate, nitrocellulose, adipic acid/neopentyl glycol/trimellitic copolymer anhydride, acetyl tributyl citrate, isopropyl alcohol was used as a basis for the preparation of antifungal lacquer with compound 2. As a reference drug was taken terbinafine in the form of $1 \%$ nail lacquer "Lamisil". Nail lacquer without the addition of antifungal agents was used as a control.

Lacquers with derivative 2 at concentrations of $1 \%, 0.5 \%$, and $0.1 \%$ and diluted lacquer with terbinafine "Lamisil" to concentrations of $0.5 \%$ and $0.1 \%$ were prepared for the study. Lacquers with appropriate concentrations, a control sample of the nail lacquers "Nogotok" and "Lamisil" were added to microscope slides, placed in Petri dishes, and covered with agarized sterile medium under laminar conditions. Then the test culture of fungus $A$. niger was added, and the dishes were cultured at $28 \pm 1^{\circ} \mathrm{C}$ in the thermostat. The results of the observations were recorded after $24 \mathrm{~h}$ (Figure 1) and $72 \mathrm{~h}$ (Figure 2). 
After $24 \mathrm{~h}$ incubation (Figure 1) in dishes with derivative 2 at a concentration of $0.1 \%$, a fungicidal effect was observed with a small number of fungal colonies compared to the control, while terbinafine at the same concentration was slightly predominant. Compound 2 at a concentration of $0.5 \%$ caused the same growth of the mycelium of the fungus $A$. niger as in control, whereas under the action of terbinafine, the amount of mycelium was higher. Amino acid derivative 2 at a concentration of $1 \%$ had a fungicidal effect at the same concentration of terbinafine, whereas the control contained a lot of fungal mycelium.

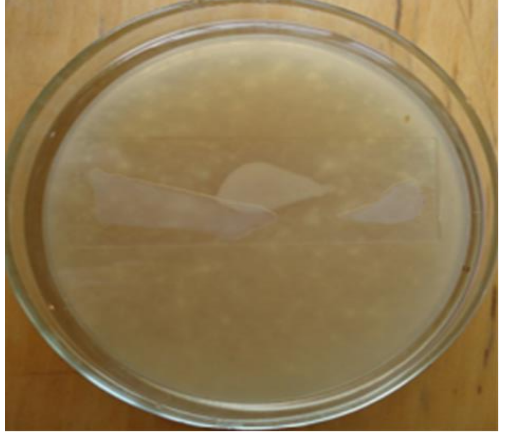

control

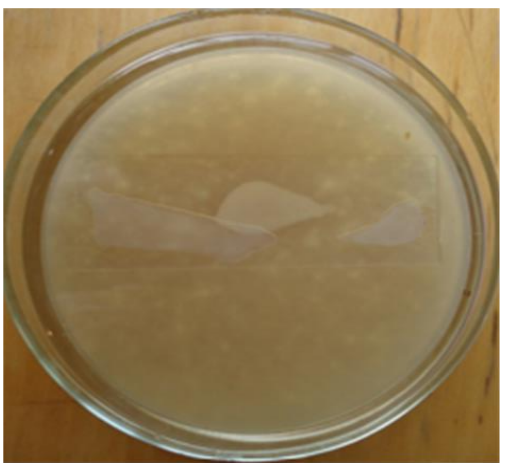

control

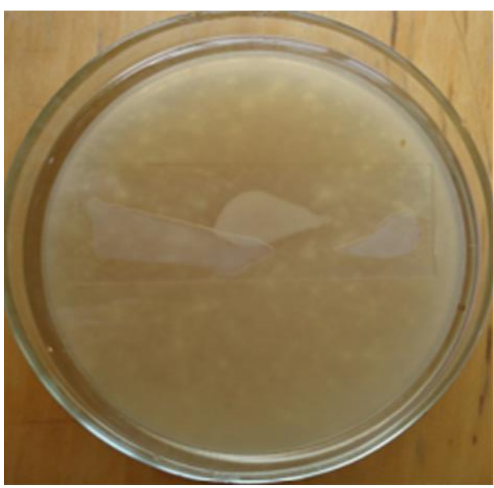

control

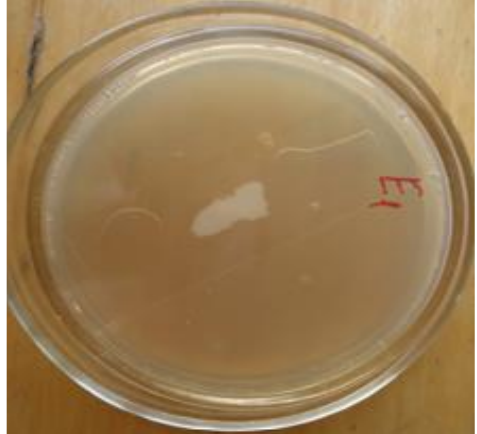

$0.1 \%$ terbinafine

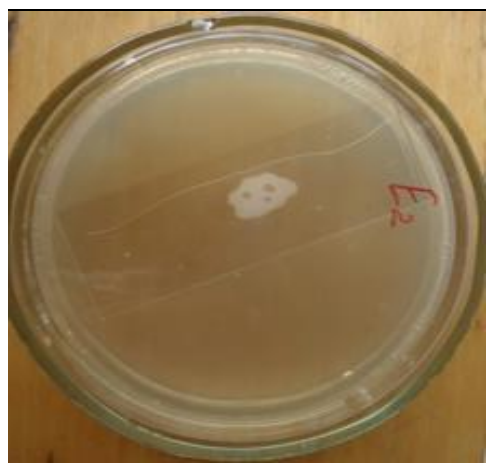

$0.5 \%$ terbinafine

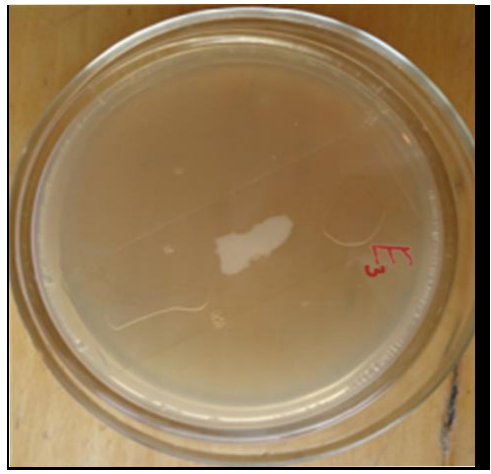

$1 \%$ terbinafine

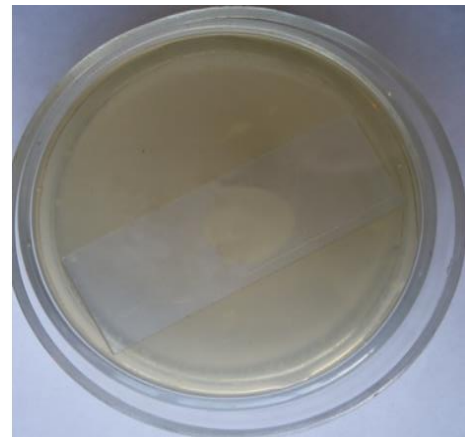

$0.1 \%$ compound 2

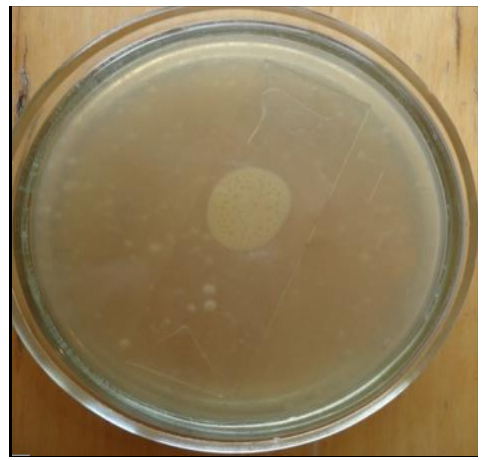

$0.5 \%$ compound 2

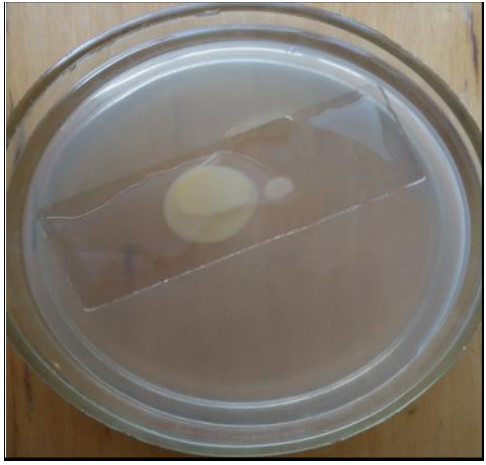

$1 \%$ compound 2

Figure 1. The influence of derivative 2 and terbinafine in the concentrations of $0.1,0.5$, and $1 \%$ in the form of lacquer on the growth of the test culture of the fungus A. niger compared to the control after $24 \mathrm{~h}$.

The results of observations after $72 \mathrm{~h}$ of incubation of the test culture (Figure 2) showed that the control sample was completely overgrown with spores of the test culture of the fungus A. niger. The compound 2 at a concentration of $0.1 \%$ showed only fungistatic activity, while $0.1 \%$ terbinafine had a fungistatic and fungicidal effect. Derivative 2 and terbinafine at a concentration of $0.5 \%$ had almost the same effect as in the case of $0.1 \%$ concentration. GABA 
derivative 2 only in the case of $1 \%$ concentration caused a zone of inhibition of growth of $A$. niger with a diameter of $22 \mathrm{~mm}$ and a smaller number of fungal spores.

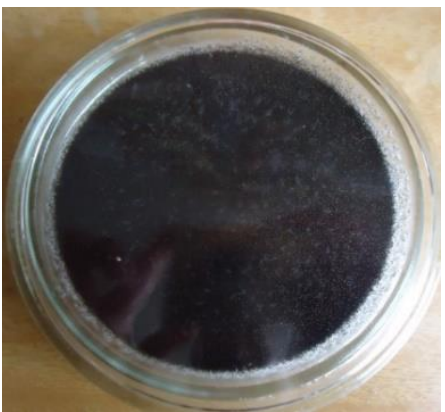

control

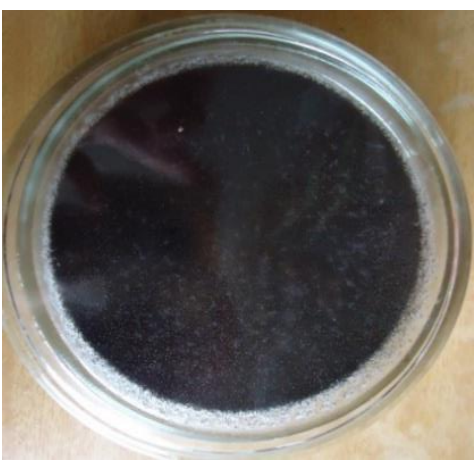

control

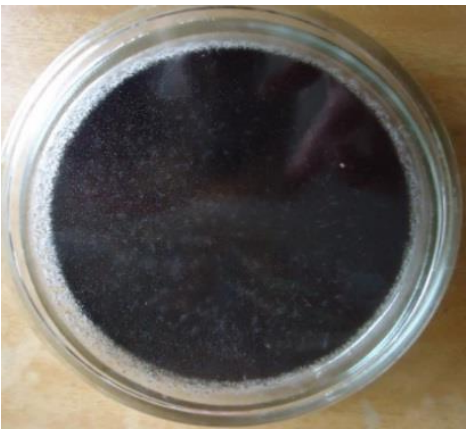

control

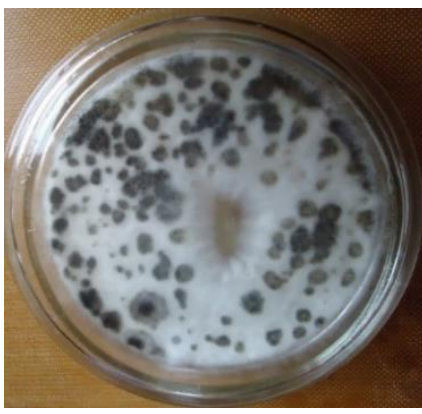

$0.1 \%$ terbinafine

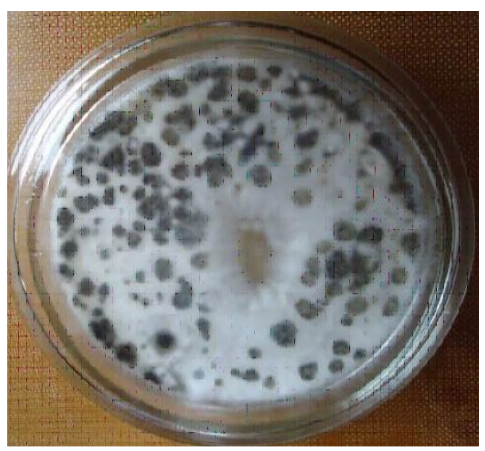

$0.5 \%$ terbinafine

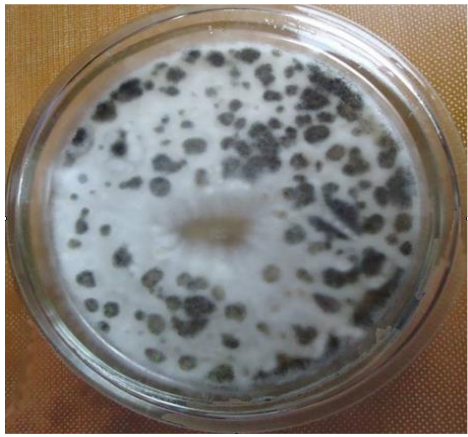

$1 \%$ terbinafine

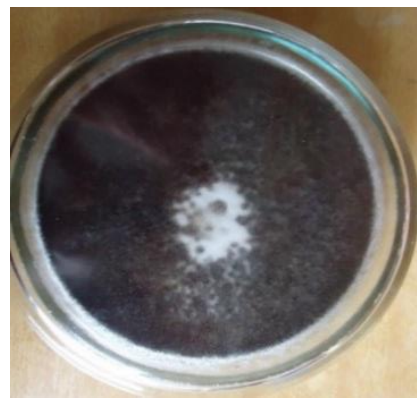

$0.1 \%$ compound 2

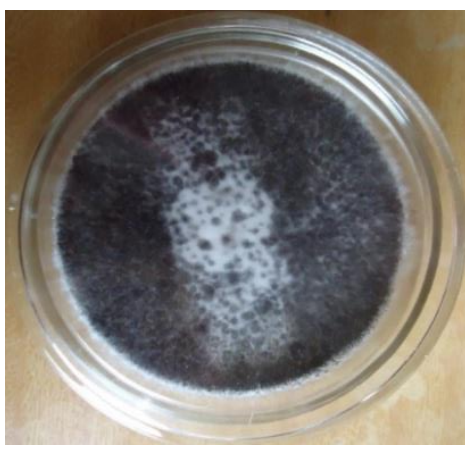

$0.5 \%$ compound 2

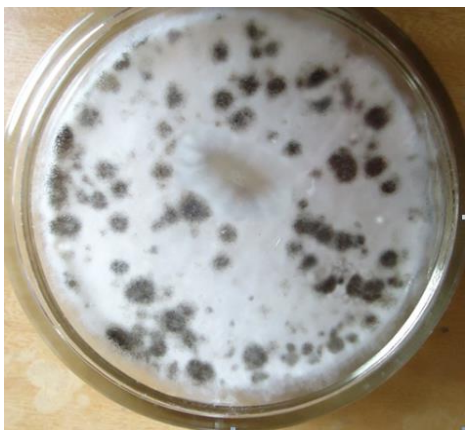

$1 \%$ compound 2

Figure 2. The influence of derivative 2 and terbinafine in the concentrations of $0.1,0.5$, and $1 \%$ in the form of lacquer on the growth of the test culture of the fungus A. niger compared to the control after $72 \mathrm{~h}$.

The reference drug at a concentration of $1 \%$ showed a slightly smaller $(20 \mathrm{~mm})$ zone of growth inhibition and a higher growth of test culture spores. But in both cases (derivative 2 and terbinafine) a concentration of $1 \%$ caused fungicidal and fungistatic action of lacquer.

Thus, the results of the study of derivative 2 in the form of the lacquer, both after $24 \mathrm{~h}$ and after $72 \mathrm{~h}$ indicate the prospects for further research in this direction.

\section{Conclusions}

Therefore, we carried out obtaining the antifungal lacquer based on the GABA derivative of 2-chloro-N-(9,10-dioxo-9,10-dihydroanthracen-1-yl)acetamide as a perspective antifungal agent against fungus strain A. niger. Study of the antifungal action of obtained lacquer at concentrations of $1 \%, 0.5 \%$, and $0.1 \%$ of active derivative 2 in comparison with the known antifungal lacquer "Lamisil" in the same concentrations showed that $4-((2-)(9,10-$ 
dioxo-9,10-dihydroanthracen-1-yl)amino)-2-oxoethyl)amino)butanoic acid $\mathbf{2}$, as well as terbinafine at a concentration of $1 \%$, has fungicidal and fungistatic action. The obtained results indicate the prospects of further research in the direction of preparation of the antifungal lacquer containing derivatives of 9,10-anthracenedione.

\section{Funding}

This research was funded by the Ministry of Education and Science of Ukraine, Project number: 0119U002252.

\section{Acknowledgments}

This research has no acknowledgment.

\section{Conflicts of Interest}

The authors declare no conflict of interest

\section{References}

1. Gupta, A.K.; Stec, N. Recent advances in therapies for onychomycosis and its management. F1000Research 2019, 8, https://doi.org/10.12688/f1000research.18646.1.

2. Aggarwal, R.; Targhotra, M.; Kumar, B.; Sahoo, P.K.; Chauhan, M.K. Treatment and management strategies of onychomycosis. J. Mycol. Med. 2020, 30, https://doi.org/10.1016/j.mycmed.2020.100949.

3. Monod, M.; Méhul, B. Recent Findings in Onychomycosis and Their Application for Appropriate Treatment. J. Fungi 2019, 5, 20, https://doi.org/10.3390/jof5010020.

4. Phaitoonwattanakij, S.; Leeyaphan, C.; Bunyaratavej, S.; Chinhiran, K. Trichophyton erinacei Onychomycosis: The First to Evidence a Proximal Subungual Onychomycosis Pattern. Case Rep. Dermatol. 2019, 11, 198-203, https://doi.org/10.1159/000501424.

5. Tamer, F.; Yuksel, M.E. Onychomycosis due to mixed infection with non-dermatophyte molds and yeasts. Our Dermatology Online 2019, 10, 267-269, https://doi.org/10.7241/ourd.20193.10.

6. Verma, K.; Tegta, G.R.; Verma, G.; Verma, S. A rare case of onychomycosis due to Aspergillus species in a neonate. Indian Journal of Paediatric Dermatology 2019, 20, 249, https://doi.org/10.4103/ijpd.IJPD_116_18.

7. Mehta, M.; Sharma, J.; Bhardwaj, S.B. Proximal subungual onychomycosis of digitus minimus due to Aspergillus brasiliensis. The Pan African Medical Journal 2020, 35, https://doi.org/10.11604/pamj.2020.35.79.20762.

8. Borgohain, P.; Barua, P.; Dutta, P.J.; Shaw, D.; Rudramurthy, S.M. Onychomycosis Associated with Superficial Skin Infection Due to Aspergillus sydowii in an Immunocompromised Patient. Mycopathologia 2019, 184, 683-689, https://doi.org/10.1007/s11046-019-00383-2.

9. Yadav, K. A review article on Anti-Fungal Nail Lacquer Using Treatment of Onychomycosis. International Journal of Scientific and Research Publications. 2019, https://doi.org/10.29322/IJSRP.9.04.2019.p8848.

10. Vikas, A.; Rashmin, P.; Mrunali, P.; Chavan, R.B.; Kaushik, T. Mechanistic Insights of Formulation Approaches for the Treatment of Nail Infection: Conventional and Novel Drug Delivery Approaches. AAPS PharmSciTech 2020, 21, 67, https://doi.org/10.1208/s12249-019-1591-9.

11. Akhtar, N.; Sharma, H.; Pathak, K. Onychomycosis: potential of nail lacquers in transungual delivery of antifungals. Scientifica 2016, 2016, https://doi.org/10.1155/2016/1387936.

12. Yau, M.; Soni, A.; Siu, W. L. How to treat fungal nail effectively. The Pharmaceutical Journal, 2018, 301, 7919, https://doi.org/10.1211/PJ.2018.20205630.

13. Šveikauskaite, I.; Briedis, V. Potential of Naftifine Application for Transungual Delivery. Molecules. 2020, 25, 3043, https://doi.org/10.3390/molecules25133043.

14. Sigurgeirsson, B.; Ghannoum, M.A.; Osman-Ponchet, H.; Kerrouche, N; Sidou, F. Application of cosmetic nail varnish does not affect the antifungal efficacy of amorolfine 5\% nail lacquer in the treatment of distal subungual toenail onychomycosis: results of a randomised active-controlled study and in vitro assays. Mycoses 2016, 59: 319-326, https://doi.org/10.1111/myc.12473.

15. Lohani, B.; Kumar, G. Medicated nail lacquers - for effective treatment of nail disorders. Indo Am. J. Pharm. Sci. 2018, 5, 1392-1403, https://doi.org/10.5281/zenodo.1197498. 
16. Ghannoum, M.; Long, L.; Kunze, G.; Sarkany, M.; Osman-Ponchet, H. A pilot, layerwise, ex vivo evaluation of the antifungal efficacy of amorolfine 5\% nail lacquer vs other topical antifungal nail formulations in healthy toenails. Mycoses 2019, 62, 494-501, https://doi.org/10.1111/myc.12896.

17. Laubé, F.; Poupon, A.; Zinck, P.; Müller-Goymann, C.; Reichl, S.; Nardello-Rataj, V. Physicochemical investigations of native nails and synthetic models for a better understanding of surface adhesion of nail lacquers. Eur. J. Pharm. Sci. 2019, 131, 208-217, https://doi.org/10.1016/j.ejps.2019.02.014.

18. Chessa, M.A.; Iorizzo, M.; Richert, B.; López-Estebaranz, J.L.; Rigopoulos, D.; Tosti, A.; Gupta, A.K.; Di Chiacchio, N.; Di Chiacchio, N.G.; Rubin, A.I.; Baran, R.; Lipner, S.R.; Daniel, R.; Chiheb, S.; Grover, C.; Starace, M.; Piraccini, B.M. Pathogenesis, Clinical Signs and Treatment Recommendations in Brittle Nails: A Review. Dermatology and Therapy 2020, 10, 15-27, https://doi.org/10.1007/s13555-019-00338-x.

19. Madnani, N.A.; Khan, K.J. Nail cosmetics. Indian Journal of Dermatology, Venereology, and Leprology 2012, 78, 309, https://doi.org/10.4103/0378-6323.95445.

20. Malik, E.M.; Müller, C.E. Anthraquinones as pharmacological tools and drugs. Med. Res. Rev. 2016, 36, 705-748, https://doi.org/10.1002/med.21391.

21. Mohamadzadeh, M.; Zarei, M.; Vessal, M. Synthesis, in vitro biological evaluation and in silico molecular docking studies of novel $\beta$-lactam-anthraquinone hybrids. Bioorg. Chem. 2020, 95, 103515, https://doi.org/10.1016/j.bioorg.2019.103515.

22. Zvarich, V. I.; Stasevich, M. V.; Stan'ko, O. V.; Komarovskaya-Porokhnyavets, E. Z.; Poroikov, V. V.; Rudik, A. V.; Lagunin, A. A.; Vovk, M. V.; Novikov, V. P. Computerized prediction, synthesis, and antimicrobial activity of new aminoacid derivatives of 2-chloro-N-(9,10-dioxo-9,10-dihydroanthracen-1yl)acetamide. Pharm. Chem. J. 2014, 48, 582-586. https://doi.org/10.1007/s11094-014-1154-z.

23. Stasevych, M.; Zvarych, V.; Lunin, V.; Kopak, N.; Komarovska-Porokhnyavets, O.; Deniz, N.G.; Sayil, C.; Ozyurek, M.; Guclu, K.; Vovk, M.; Novikov, V. Synthesis and investigation of antimicrobial and antioxidant activity of anthraquinonylhydrazones. Monatshefte für Chemie - Chemical Monthly 2018, 149, 1111-1119, https://doi.org/10.1007/s00706-018-2157-3.

24. Zvarych, V.; Stasevych, M.; Novikov, V.; Rusanov, E.; Vovk, M.; Szweda, P.; Grecka, K.; Milewski, S. Anthra[1,2- $d][1,2,3]$ triazine-4,7,12(3H)-triones as a new class of antistaphylococcal agents: synthesis and biological evaluation. Molecules 2019, 24, 4581. https://doi.org/10.3390/molecules24244581.

25. Stasevich, M.V.; Zvarich, V.I.; Novikov, V.P.; Zagorodnyaya, S.D.; Povnitsa, O.Y.; Chaika, M.A.; Nesterkina, M.V.; Kravchenko, I.A.; Druzhilovskii, D.S.; Poroikov, V.V. 9,10-Anthraquinone Dithiocarbamates as Potential Pharmaceutical Substances with Pleiotropic Actions: Computerized Prediction of Biological Activity and Experimental Validation. Pharm. Chem. J. 2020, 53, 905-913, https://doi.org/10.1007/s11094-020-02098-x.

26. Aryandi, R.; Agustin Krisna, W.; Chi, I.C. Anthraquinone Derivatives and its Antibacterial Properties from Paederia foetida Stems. The Natural Products Journal 2020, 10, 1-7, https://doi.org/10.2174/2210315510666191224103057.

27. Armarego, W. L. F.; Chai, C. Purification of Laboratory Chemicals, 7th ed.; Elsevier: Oxford, UK, 2013, https://doi.org/10.1016/C2009-0-64000-9.

28. NCCLS. Reference method for broth dilution antifungal susceptibility testing of conidium forming filamentous fungi: proposed standard, document M38-P; NCCLS: Wayne, PA, USA, 1998.

29. Stasevych, M.; Zvarych, V.; Lunin, V.; Halenova, T.; Savchuk, O.; Dudchak, O.; Vovk, M.; Novikov, V. Novel anthraquinone-based derivatives as potent inhibitors for receptor tyrosine kinases. Indian J. Pharm. Sci. 2015, 77, 634, https://doi.org/10.4103/0250-474x.169062

30. Zvarych, V.; Stasevych, M.; Lunin, V.; Deniz, N.G.; Sayil, C.; Ozyurek, M.; Guclu, K.; Vovk, M.; Novikov, V. Synthesis and investigation of antioxidant activity of the dithiocarbamate derivatives of 9,10anthracenedione. Monatshefte für Chemie - Chemical Monthly 2016, 147, 2093-2101, https://doi.org/10.1007/s00706-016-1839-y

31. Stasevych, M.V.; Zvarych, V.I.; Stan'ko, O.V.; Vovk, M.V.; Novikov, V.P. Synthesis of 2-(NBenzoylimino)-N-(9,10-Dioxo-9,10-Dihydroanthracen-1-yl)Thiazoles. Chemistry of Heterocyclic Compounds 2014, 49, 1831-1833, https://doi.org/10.1007/s10593-014-1437-z.

32. Zvarych, V.I.; Stasevych, M.V.; Lunin, V.V.; Vovk, M.V.; Novikov, V.P. Synthesis of (1H-pyrrol-1yl)anthracene-9,10-diones. Chemistry of Heterocyclic Compounds 2016, 52, 421-423, https://doi.org/10.1007/s10593-016-1904-9.

33. Stasevych, M.; Zvarych, V.; Musyanovych, R.; Novikov, V.; Vovk, M. Synthesis of N-Benzoyl-N'-(9, 10Dioxo-9, 10-Dihydroanthacen-1-yl) Thioureas and Quantum-Chemical Analysis of the Reaction Passing. Chemistry \& Chemical Technology 2014, 135-140, https://doi.org/10.23939/chcht08.02.135. 